\title{
Normal Zone Propagation in Superconducting Focusing Solenoids and Related Quench Protection Issues
}

\author{
I. Terechkine and V. Veretennikov
}

\begin{abstract}
Superconducting solenoids are increasingly used as focusing lenses in transport channels of proton linear RF accelerators. If these accelerators employ superconducting RF cavities, each focusing lens is usually comprised of three coils connected in series: a main coil, which provides the needed focusing strength, and two bucking coils, that help to reduce magnetic field outside the lens.

When a normal zone develops in any of the coils in a focusing lens, it propagates with a direction and a rate which depends on the coil and the specific part of the coil in which the quench first occurred. As a result of this propagation process (quenching), the temperature and/or voltage of parts of the lens can exceed safe limits, thus compromising lens reliability. On the other hand, the negative impact of quench events can be significantly mitigated if an external resistor is used to absorb a part of the energy stored in the magnetic field.

This paper presents the main results of a solenoid quench protection study based on computational modeling of normal zone propagation in solenoid lenses being built for a superconducting linear RF accelerator under development at Fermilab.
\end{abstract}

Index Terms - accelerator magnets, protection, solenoids, superconducting linear accelerators, superconducting magnets.

\section{INTRODUCTION}

$\mathrm{T}$ limit emittance growth in low energy sections of high current linear ion accelerators, focusing solenoids are often used [1]. If RF superconducting structures are used for acceleration, the focusing lens, which is usually located between accelerating cavities, is often comprised of a main coil and two bucking coils that are used to reduce the fringe field of the lens to the level that permits further shielding with the use of magnetically soft alloys, as it was suggested in [2] and [3]. The front end of an $\mathrm{H}^{-} \mathrm{RF}$ accelerator under development at FNAL for the High Intensity Neutrino Source (HINS) project makes use of three types of accelerating sections that employ solenoids for beam focusing [4]. The socalled "CH" section is a conventional, DTL-type, room temperature accelerating structure with focusing lenses installed with $\sim 0.5 \mathrm{~m}$ period. Two superconducting RF

Manuscript received August 24, 2007.

This work was supported by the U.S. Department of Energy under contract No. DE-AC02-07CH11359.

Authors are with the Fermi National Accelerator laboratory, Batavia, IL 60510 USA (630-840-4017; fax: 630-840-8036; e-mail: terechki@fnal.gov). sections that follow the $\mathrm{CH}$ section (SS-1 and SS-2) use "spoke cavities", optimized for the specific range of beam particle velocities, for acceleration. Although all three sections use solenoid-based focusing lenses, the required focusing strength and lens bore diameter is different for each section. The number of solenoids in the front end if HINS linac is quite high ( 50 in the case described in [4]), so reliability of the focusing system becomes a concern. As shown in [5], the main source of uncertainty is the temperature and voltage developed in a bucking coil during quenching. In this case, the energy, mainly stored in the magnetic field of the main coil of the device, dissipates in the quenching bucking coil, which is quite small compared to the main coil. The maximum temperature in the bucking coil approaches $90 \mathrm{~K}$ and the voltage in the coil reaches $330 \mathrm{~V}$ with a $1 \mathrm{Ohm}$ dump resistor in the discharge circuit used to dissipate part of the stored energy. Without using a dump resistor, the temperature in the coil would reach $180 \mathrm{~K}$ and the electric potential would approach $500 \mathrm{~V}$.

The focusing strength of solenoids for SS-1 and SS-2 sections of the HINS accelerator is higher than that of the solenoids used in the $\mathrm{CH}$ section and, since the volume of the bucking coils remains almost the same, one should expect higher temperature and voltage in quenching bucking coils in these sections. By studying quench propagation and related solenoid protection issues for these systems, we can identify problems and choose appropriate solutions.

When carrying out the quench protection analysis for the $\mathrm{CH}$ section of the HINS linac, we used "home-made" Matlabbased software [6] that allowed visualization of the temperature and voltage to ground at any point inside the solenoid as a function of time. A provision for modeling a dump resistor was also included in the program. Significant drawbacks of this program included relatively low performance (several hours of computing time for a typical case) and the absence of any interface. After rewriting the software using a different computational approach to improve the performance and add more flexibility to the code, a study has been made of quench-related effects in focusing solenoids of the first superconducting RF section (SS-1) of the HINS linac front end. This paper presents main results of this study and discusses some features of the code. 


\section{Design Features of A Focusing SOlEnoID FOR SS-1}

There are two types of focusing solenoids in each section of the front end of the HINS accelerator: with and without steering dipoles. From the viewpoint of quench propagation and protection, there is only a slight difference between the two types of the solenoids; for the purpose of this paper, only solenoids for SS-1 section of HINS without dipole correctors will be discussed. The schematic view in Fig. 1 displays the longitudinal cross-section of the solenoid. Details of the solenoid design can be found in [7]. Bucking coils (BC1 and $\mathrm{BC} 2$ ) are placed near the ends of the main coil (MC). NbTi strand is used to wind the coils and each coil is wound on a bobbin made of G-10. After the coils are wound, they are insulated using fiberglass tape and then impregnated with epoxy. All the coils are connected in series with the magnetic field of the BC's directed opposite to that of the MC. For the purpose of the quench propagation study, we will assume that there is liquid Helium everywhere outside the coils. The geometric and winding parameters of the coils are also found in Fig. 1.

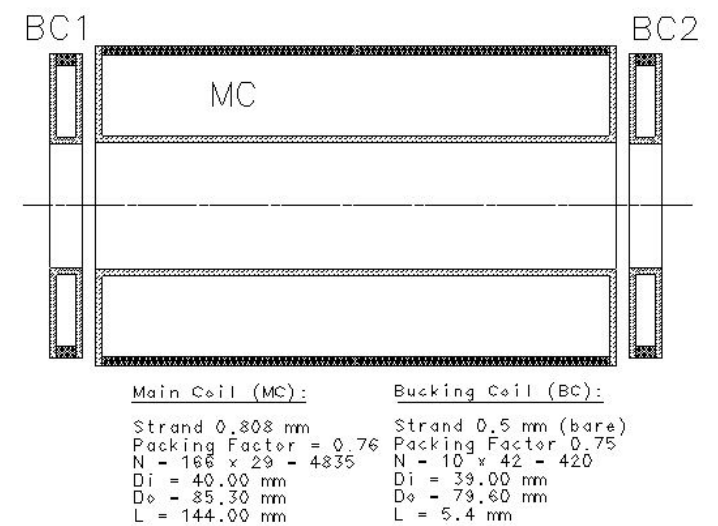

Fig. 1. Schematic of a solenoid used for modeling quench propagation.

The increased stored energy of the solenoids in SS-1 (compared with the solenoids of the $\mathrm{CH}$ section of the HINS linac) results in a higher voltage developed in the system during quenching and a higher final temperature in a quenching bucking coil. The next section presents main features of quench propagation for the SS-1 solenoid.

\section{QUenCh PROTECTION STUDY}

There are two requirements to the focusing solenoid that must be met to prevent an irreversible, quench-related failure: the temperature inside the coil should not exceed $\sim 300 \mathrm{~K}$ and the maximum voltage in the coil during quenching must not exceed what is used during the solenoid acceptance test. The allowed voltage depends on the insulation system used. In the case of the focusing solenoids for the HINS linac front end, the requirement of a high compaction factor in the coils (see [3] for details) results in relatively weak insulation, so $1000 \mathrm{~V}$ was chosen for the room temperature insulation quality ("hipot") test and $500 \mathrm{~V}$ was used for testing the system at $4.2 \mathrm{~K}$. The maximum temperature requirement is set to limit the thermal stress that the coils experience due thermal gradients during quenching. This value can be adjusted after results of more tests are available, but in our case, at this point, we accept the $300 \mathrm{~K}$ limit. The temperature gradients in quenching coil depend on where quench starts and the quench current. It has been found for the epoxy-impregnated coils of the SS-1 solenoid that, the maximum temperature is higher for higher current and only weakly depends on the location of the initial quench point [8]. Two distinctive cases were studied: without and with a dump resistor in the current discharge circuit.

\section{A. Without a Dump Resistor}

Fig. 2 shows temperature distribution in the bucking coil in the end of the discharge cycle when quench was initiated in the area of lowest magnetic field region, which corresponds to turn \#1 in layer \#23. The maximum temperature reaches 255 $\mathrm{K}$ in the $6^{\text {th }}$ turn of the $23^{\text {rd }}$ layer, which is closer to the point of the maximum magnetic field point.

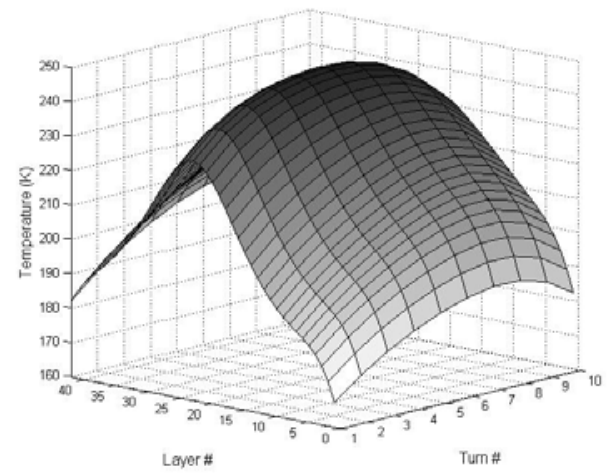

Fig. 2. Temperature distribution in the bucking coil after quenching. Quench start point is $\mathrm{l}=23, \mathrm{t}=1 ; \mathrm{I} 0=200 \mathrm{~A}$.

In the case when quench is initiated in the highest field region (turn \#10 in layer \#23), the maximum temperature reaches $260 \mathrm{~K}$ and is localized in the turn $8-9$ region.

The value and location of the maximum voltage in the coil, besides being dependent on the initial current and quench start location, also depends on the details of the current discharge circuit and distribution of the normal zone in the body of the quenching coil at any moment during quenching. As in the case of the $\mathrm{CH}$ section solenoid in [1], the worst-case scenario takes place when the quench starts in one of the bucking coils in the maximum magnetic field region. Faster quench propagation in this area leads to faster current decay and corresponding higher inductive voltage generated by the main coil. The location of the voltage maximum is between the main coil and the quenching bucking coil. The graph in Fig. 3 shows how voltage at this point changes with time.

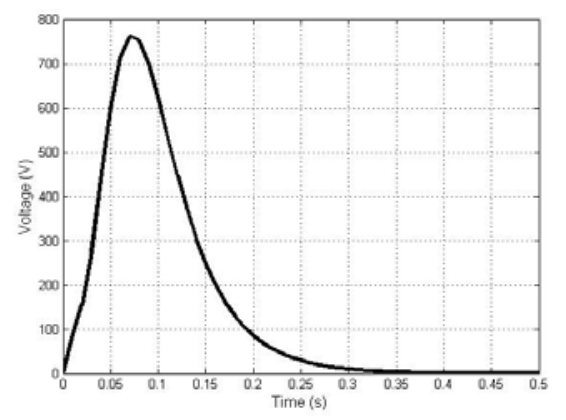

Fig. 3. Electrical potential in the outer layer of the bucking coil during quenching. Quench start point is $\mathrm{l}=23, \mathrm{t}=10 ; \mathrm{I} 0=200 \mathrm{~A}$. 
In this case, the maximum voltage reaches $\sim 760 \mathrm{~V}$, which is not acceptable; a way must be found to bring the voltage in the solenoid below the $500 \mathrm{~V}$ limit.

\section{B. Dump Resistor in the Current Discharge Circuit}

Connecting a dump resistor in series in the current discharge circuit with some delay or immediately after detection of a quench helps to reduce the maximum voltage developed during a quench. It was shown in [1] that in order to decrease the maximum voltage in the solenoid, the dump resistor should be connected between the main coil and ground. With this setting, the worst-case scenario is when a quench starts in the high field part of the grounded bucking coil. The graph in Fig. 4 demonstrates how the maximum temperature in the quenching bucking coil depends on the value of the dump resistor. This temperature depends on what fraction of the stored energy is dissipated in the resistor. For example, with $\mathrm{R}_{\text {dump }}=2 \mathrm{Ohm}$, more than $50 \%$ of the stored energy can be dissipated externally.

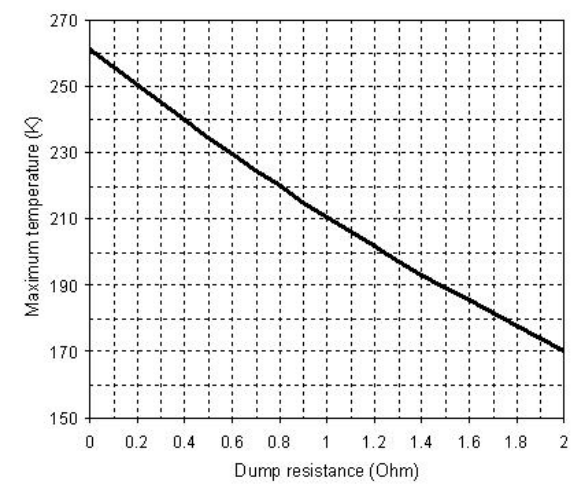

Fig. 4. Maximum temperature in the bucking coil as a function of a dump resistor value. Quench initiation point is $\mathrm{l}=23, \mathrm{t}=10$; $\mathrm{I} 0=200$.

By analyzing the voltages at different points of the discharge circuit, one can determine an optimal value of the dump resistor. The initial voltage on the dump resistor reaches its maximum value at the moment the resistor is connected: $\mathrm{U}_{\mathrm{Rd}}=\mathrm{I} 0 \cdot \mathrm{R}_{\mathrm{dump}}$. Resistance growth in the quenching coil (the value of this resistance can be higher than that of the dump resistance) is responsible for the appearance of the resistive component of voltage inside the coil: $\mathrm{U}_{\mathrm{c}}=\mathrm{I}(\mathrm{t}) \cdot \mathrm{R}_{\mathrm{c}}(\mathrm{t})$. The inductive component of the voltage depends on the initial current $\mathrm{I}_{0}$ and on the dynamics of the current decay, which is defined by the total resistance in the circuit. As a result, the maximum voltage, which is a sum of the resistive and inductive components, can have different signs and its absolute value can exceed $\left|\mathrm{U}_{\mathrm{Rd}}\right|$. In the case of the SS-1 solenoid, the value of the dump resistor that corresponds to the minimal value of the maximum voltage is $\sim 1.9 \mathrm{Ohm}$. At $\mathrm{I}_{0}=$ $200 \mathrm{~A}$ and $\mathrm{R}_{\text {dump }}$ between 1.8 and $2.0 \mathrm{Ohm}$, the voltage at any point in the system does not exceed $400 \mathrm{~V}$. If $\mathrm{R}_{\text {dump }}<1.2 \mathrm{Ohm}$ or $\mathrm{R}>2.5 \mathrm{Ohm}$, the maximum voltage exceeds $500 \mathrm{~V}$.

\section{Other methods of Quench Protection}

As we have seen, even with the use of a dump resistor, the maximum voltage is quite high, although below the allowable limit. If focusing solenoids with higher focusing strength are needed (e.g. focusing solenoids for the SS-2 section of HINS), there could be a problem in finding a reasonable solution using only a dump resistor. Another common method of quench protection using quench heaters was studied in [8], but the results were not satisfactory: neither the temperature, nor the voltage changed much after quench heaters were introduced at different locations inside bucking coils.

A more radical, and probably more expensive, protection measure is to use a separate power supply to power the bucking coils.

\section{A Code For Modeling Quench Propagation}

A large number of different parameters - including the geometry of the coils, strand critical surface, winding density, thermal properties of epoxy-impregnated coil structure, and the quench current, must be taken into account for a thorough analysis of all quench-related effects. This requires a proper tool. At different times, in different laboratories, and by different people, for different superconducting magnetic systems, varieties of codes for quench analysis have been developed (e.g., see [9], [10]). Some of these codes were not universal enough to provide a convenient environment suitable for our goals; others were not immediately available for installation and use. Commercial programs for making quench analysis also exist, but those we are familiar with have a price tag that is out of our reach. The relatively simple geometry of focusing solenoids and the well defined physics of normal zone propagation, permitted us to develop our own code, which was used to get the results presented above. Algorithm used during modeling is shown in Fig. 5 below.

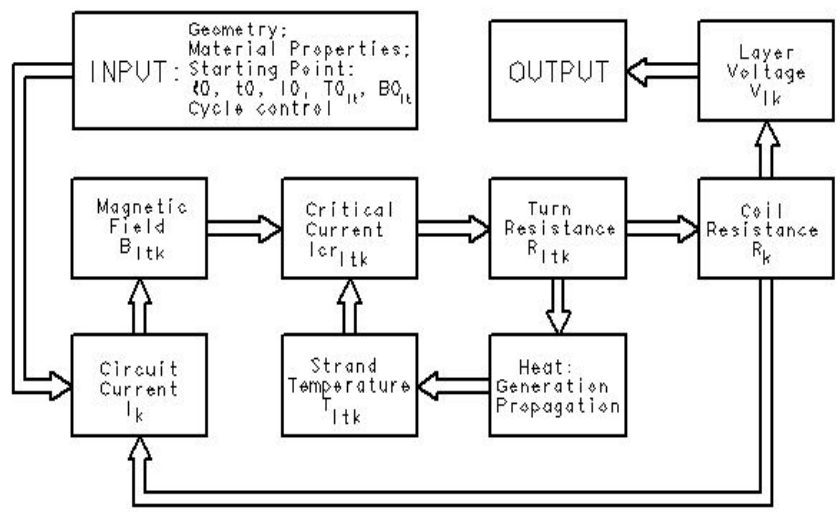

Fig. 5. Algorithm used to model quench propagation in focusing solenoids.

\section{A. Algorithm Description}

Input blocks of the program are used to define the geometry of the solenoid to be modeled and a set of relevant material properties: specific heat, specific resistance, thermal conductivity, and the critical surface of a superconductor. The magnetic field distribution in the coils of a solenoid can be found using any magnetic modeling program and is presented as an array of a convenient size, which is interpolated by the program. The initial current and temperature distribution inside the quenching coil define the initial conditions of a particular quench process. The inductances of coils in solenoids are calculated, after taking into account its geometry, by using convenient handbook approximations for 
mutual inductances of turns. This simplifies the calculation of voltages in the coil.

An effective rectangular strand was introduced to replace round strand that was usually used in the real coil. The crosssection of the effective strands and effective inter-layer and inter-turn insulation thicknesses were defined in the input block based on the strand and coil winding parameters for each coil.

A set of control parameters is used to adjust time scale of the process and details of how the output data are stored.

All the data are stored as 3-D arrays to reflect, for every turn in the coil for any moment in time, temperature and local material properties, that depend on temperature and, in the cases of the strand specific resistance and critical current, on magnetic field.

The modeling process starts by comparing the initial temperature array with the array of the critical temperatures at the location of each turn. If local temperature is above the critical point, the turn resistance is evaluated, followed by calculation of heat deposition and heat propagation to neighboring turns through thermal conductance, which can differ in the radial and longitudinal directions. Temperature rise of the neighboring turns is defined by the heat capacity of the winding (both strand and insulation are taken into the account). Knowing the critical surface of a superconductor, one can find the time corresponding to transition to the normal state for any turn.

To simplify the task, only a 2D problem is solved; this means that heat transfer along the strand is neglected and the propagation is only in the transverse direction (radially and longitudinally). As was shown in [11], if the coil is impregnated with epoxy or another media with good thermal conductivity, as it is in our case, heat transfer along the strand, although certainly playing a role, is significantly less than the transverse heat propagation.

At each time step, the arrays of heat deposition, temperature rise, critical current, and resistance for each turn are recalculated; the sum of resistances of all the turns gives the updated value of the coil resistance. Knowing the inductances and resistances of the coils and other elements in the current discharge circuit, the current drop can be calculated and the value of the current updated. This results in an updated magnetic field map and the process repeats itself by iterating until instructed to stop.

\section{B. Code Upgrade}

By using the earlier developed code described in [6], we could study quench propagation and related protection issues for the focusing solenoid of the CH section of HINS linac [5]. Slow performance and the absence of a convenient interface prompted us to rewrite the code using an object-oriented approach and vector operations. A typical run now takes just several minutes to finish.

Several important options have been added to the program:

- It is now possible to add several quench heaters to the system with controlled timing of firing;

- $\quad$ Timing of the dump resistor insertion in the circuit can now be set and changed arbitrarily;
User's interface to the code is still under development; when finished, it will allow interactive change of the input and control parameters adding significant flexibility for users.

\section{CONCLUSION}

A code for quench-related studies in focusing solenoids has been developed at FNAL. Analysis of quench protection for the SS-1 focusing solenoid of HINS linac made with the use of this code provided us with details of the system behavior after quenching. It has been shown that a dump resistor must be used to protect the SS-1 solenoid from possible failure of the ground insulation during quenching.

Modification of the code by adding additional options for modeling made the code a convenient tool for modeling quench propagation in focusing solenoids. The immediate application of the code will be analysis of a solenoid designed for the SS-2 section of HINS linac.

\section{ACKNOWLEDGMENT}

Both authors are very pleased to have been able to participate in Fermilab's summer employment program for students majoring in Physics, which allowed them to enjoy working on this problem and which otherwise would not have been addressed this year.

\section{REFERENCES}

[1] I. Terechkine, V. V. Kashikhin, T. Page, M. Tartaglia, and J. Tompkins, "Focusing Solenoid for the Front End of a Linear RF Accelerator", PAC-07, paper \# MOPAS019.

[2] M. Johnson, et al., "Cryomodule design for a superconducting linac with quarter-wave, half-wave, and focusing elements," PAC-05, Proceedings, pp. 4317-4319, 2006.

[3] G. Davis, V.V. Kashikhin, T. Page, I. Terechkine, J. Tompkins, and T. Wokas, "Designing focusing solenoids for superconducting RF accelerators,” IEEE Trans. Appl. Supercond., vol. 17, no. 2, June 2007, pp. 1221-1224.

[4] P. N. Ostroumov, K. W. Shepard, G. W. Foster, I. V. Gonin, and G. V. Romanov, "Front end design of a multi-GeV H-minus linac," PAC-05, Proceedings, pp. 3286-3288, 2006.

[5] I. Terechkine, "Quench Analysis for Focusing Solenoid of the $\mathrm{CH}$ Section of HINS Linac”, TD-06-067, FNAL, Dec. 2006.

[6] S. Obraztsov, I. Terechkine, "A Tool for Modeling Quench Propagation in Superconducting Focusing Solenoids and Related Protection Issues," TD-06-063, FNAL, 2006.

[7] G. Davis, V.V. Kashikhin, T. Page, I. Terechkine, and T. Wokas, "SS-1 Section Focusing Solenoid Cold Mass Design” TD-06-041, FNAL, Aug. 2006.

[8] V. Veretennikov, I. Terechkine, "HINS Linac Front End SS-1 Focusing Solenoid Quench Protection Analysis”, TD-07-020, FNAL, Aug. 2007.

[9] M. N. Wilson, RHEL Rep. M-157, Aug. 1968.

[10] D. Hagedorn, F. Rodrigues Mateos, "Modeling of the quenching process in complex superconducting magnet systems", IEEE Trans. Magn., vol. 28, Jan. 1992, pp.366-369.

[11] I. Terechkine, P. Bauer, PD Front End Focusing Solenoid Quench Protection Studies. Pt I: Method Description and the First Iteration, TD06-003, FNAL TD, 2006 